\title{
Stability of the $n$-Dimensional Mixed-Type Additive and Quadratic Functional Equation in Non-Archimedean Normed Spaces
}

\author{
Yang-Hi Lee, ${ }^{1}$ Soon-Mo Jung, ${ }^{2}$ and Themistocles M. Rassias ${ }^{3}$ \\ ${ }^{1}$ Department of Mathematics Education, Gongju National University of Education, \\ Gongju 314-711, Republic of Korea \\ ${ }^{2}$ Mathematics Section, College of Science and Technology, Hongik University, \\ 339-701 Jochiwon, Republic of Korea \\ ${ }^{3}$ Department of Mathematics, National Technical University of Athens, Zografou Campus, \\ 15780 Athens, Greece
}

Correspondence should be addressed to Soon-Mo Jung, smjung@hongik.ac.kr

Received 19 November 2011; Accepted 23 January 2012

Academic Editor: Muhammad Aslam Noor

Copyright (C 2012 Yang-Hi Lee et al. This is an open access article distributed under the Creative Commons Attribution License, which permits unrestricted use, distribution, and reproduction in any medium, provided the original work is properly cited.

We will prove the stability of the functional equation $2 f\left(\sum_{i=1}^{n} x_{i}\right)+\sum_{1 \leq i, j \leq n, i \neq j} f\left(x_{i}-x_{j}\right)=(n+$ 1) $\sum_{i=1}^{n} f\left(x_{i}\right)+(n-1) \sum_{i=1}^{n} f\left(-x_{i}\right)$ in non-Archimedean normed spaces.

\section{Introduction}

A classical question in the theory of functional equations is "when is it true that a function, which approximately satisfies a functional equation, must be somehow close to an exact solution of the equation?" Such a problem, called a stability problem of the functional equation, was formulated by Ulam in 1940 (see [1]). In the following year, Hyers [2] gave a partial solution of Ulam's problem for the case of approximate additive functions. Subsequently, his result was generalized by Aoki [3] for additive functions and by Rassias [4] for linear functions. Indeed, they considered the stability problem for unbounded Cauchy differences. During the last decades, the stability problems of functional equations have been extensively investigated by a number of mathematicians (see [5-23]).

A non-Archimedean field is a field $\mathbb{K}$ equipped with a function (valuation) $|\cdot|: \mathbb{K} \rightarrow$ $[0, \infty)$ such that 
$\left(F_{1}\right)|r|=0$ if and only if $r=0$;

$\left(F_{2}\right)|r s|=|r||s|$

$\left(F_{3}\right)|r+s| \leq \max \{|r|,|s|\}$ for all $r, s \in \mathbb{K}$.

Clearly, it holds that $|1|=|-1|=1$ and $|n| \leq 1$ for all $n \in \mathbb{N}$.

Let $X$ be a vector space over a scalar field $\mathbb{K}$ with a non-Archimedean and nontrivial valuation $|\cdot|$. A function $\|\cdot\|: X \rightarrow \mathbb{R}$ is a non-Archimedean norm (valuation) if it satisfies the following conditions:

$$
\begin{aligned}
& \left(N_{1}\right)\|x\|=0 \text { if and only if } x=0 ; \\
& \left(N_{2}\right)\|r x\|=|r|\|x\| \text { for all } r \in \mathbb{K} \text { and } x \in X ; \\
& \left(N_{3}\right)\|x+y\| \leq \max \{\|x\|,\|y\|\} \text { for all } x, y \in X .
\end{aligned}
$$

Then $(X,\|\cdot\|)$ is called a non-Archimedean space. Due to the fact that

$$
\left\|x_{n}-x_{m}\right\| \leq \max _{m \leq i<n}\left\|x_{i+1}-x_{i}\right\| \quad(n>m)
$$

a sequence $\left\{x_{n}\right\}$ is Cauchy if and only if $\left\{x_{n+1}-x_{n}\right\}$ converges to zero in a non-Archimedean space. A complete non-Archimedean space is a non-Archimedean space in which every Cauchy sequence is convergent.

Recently, Moslehian and Rassias [24] proved the Hyers-Ulam stability of the Cauchy functional equation

$$
f(x+y)=f(x)+f(y)
$$

and the quadratic functional equation

$$
f(x+y)+f(x-y)=2 f(x)+2 f(y)
$$

in non-Archimedean normed spaces.

We now consider the $n$-dimensional mixed-type quadratic and additive functional equation

$$
2 f\left(\sum_{i=1}^{n} x_{i}\right)+\sum_{1 \leq i, j \leq n, i \neq j} f\left(x_{i}-x_{j}\right)=(n+1) \sum_{i=1}^{n} f\left(x_{i}\right)+(n-1) \sum_{i=1}^{n} f\left(-x_{i}\right),
$$

whose solution is called a quadratic-additive function.

In 2009, Towanlong and Nakmahachalasint [25] obtained a stability result for the functional equation (1.4), in which they constructed a quadratic-additive function $F$ by composing an additive function $A$ and a quadratic function $Q$, where $A$ and $Q$ approximate the odd part and the even part of the given function $f$, respectively.

In this paper, we investigate a general stability problem for the $n$-dimensional mixedtype quadratic and additive functional equation (1.4) in non-Archimedean normed spaces. 


\section{Solutions of $(1.4)$}

In this section, we prove the generalized Hyers-Ulam stability of the $n$-dimensional mixedtype quadratic and additive functional equation (1.4). Assume that $H$ is an additive group and $X$ is a complete non-Archimedean space.

For a given function $f: H \rightarrow X$, we use the abbreviations

$$
\begin{aligned}
f_{e}(x) & :=\frac{f(x)+f(-x)}{2}, \\
f_{o}(x) & :=\frac{f(x)-f(-x)}{2}, \\
A f(x, y) & :=f(x+y)-f(x)-f(y), \\
Q f(x, y) & :=f(x+y)+f(x-y)-2 f(x)-2 f(y), \\
D_{n} f\left(x_{1}, x_{2}, \ldots, x_{n}\right) & :=2 f\left(\sum_{i=1}^{n} x_{i}\right)+\sum_{1 \leq i, j \leq n, i \neq j} f\left(x_{i}-x_{j}\right) \\
& -(n+1) \sum_{i=1}^{n} f\left(x_{i}\right)-(n-1) \sum_{i=1}^{n} f\left(-x_{i}\right)
\end{aligned}
$$

for all $x, y, x_{1}, x_{2}, \ldots, x_{n} \in H$ and for an arbitrarily fixed $n \in \mathbb{N}$.

Theorem 2.1. Assume that $n \geq 2$ is an integer. Let $H$ and $X$ be an additive group and a complete non-Archimedean space, respectively. A function $f: H \rightarrow X$ is a solution of (1.4) if and only if $f_{e}$ is quadratic, $f_{o}$ is additive, and $f_{e}(0)=0$.

Proof. If a function $f: H \rightarrow X$ is a solution of (1.4), then we have $f_{e}(0)=0$,

$$
\begin{aligned}
Q f_{e}(x, y) & =f_{e}(x+y)+f_{e}(x-y)-2 f_{e}(x)-2 f_{e}(y) \\
& =\frac{1}{2} D_{n} f_{e}(x, y, 0, \ldots, 0)+\frac{1}{2}(n-2)(n+3) f_{e}(0) \\
& =0 \\
A f_{o}(x, y) & =f_{o}(x+y)-f_{o}(x)-f_{o}(y)=\frac{1}{2} D_{n} f_{o}(x, y, 0, \ldots, 0)=0
\end{aligned}
$$

for all $x, y \in H$, that is, $f_{e}$ is quadratic and $f_{o}$ is additive.

Conversely, assume that $f_{e}$ is quadratic, $f_{o}$ is additive, and $f_{e}(0)=0$. We apply an induction on $j$ to prove $D_{n} f_{e}\left(x_{1}, x_{2}, \ldots, x_{n}\right)=0$ for all $x_{1}, x_{2}, \ldots, x_{n} \in H$. For $j=2$, we have

$$
\begin{aligned}
D_{n} f_{e} & \left(x_{1}, x_{2}, 0, \ldots, 0\right) \\
& =2 f_{e}\left(x_{1}+x_{2}\right)+2 f_{e}\left(x_{1}-x_{2}\right)-4 f_{e}\left(x_{1}\right)-4 f_{e}\left(x_{2}\right)-(n-2)(n+3) f_{e}(0) \\
& =0
\end{aligned}
$$


If $n>2$ and $D_{n} f_{e}\left(x_{1}, x_{2}, \ldots, x_{j}, 0, \ldots, 0\right)=0$ for some integer $j(2 \leq j<n)$ and for all $x_{1}, x_{2}, \ldots$, $x_{j} \in H$, then a routine calculation yields

$$
\begin{aligned}
D_{n} f_{e}( & \left.x_{1}, x_{2}, \ldots, x_{j+1}, 0, \ldots, 0\right) \\
= & Q f_{e}\left(x_{1}+\cdots+x_{j}, x_{j+1}-x_{j}\right)+\frac{1}{2} D_{n} f_{e}\left(x_{1}, \ldots, x_{j-1}, 2 x_{j}, 0, \ldots, 0\right) \\
& +\frac{1}{2} D_{n} f_{e}\left(x_{1}, \ldots, x_{j-1}, 2 x_{j+1}, 0, \ldots, 0\right)-\sum_{k=1}^{j-1}\left(Q f_{e}\left(x_{k}, x_{j}\right)+Q f_{e}\left(x_{k}, x_{j+1}\right)\right) \\
& -\frac{j}{2} Q f_{e}\left(x_{j+1}, x_{j+1}\right)-\frac{j}{2} Q f_{e}\left(x_{j}, x_{j}\right) \\
= & 0
\end{aligned}
$$

for all $x_{1}, x_{2}, \ldots, x_{j+1} \in H$. Hence, we conclude that

$$
D_{n} f_{e}\left(x_{1}, x_{2}, \ldots, x_{n}\right)=0
$$

for all $x_{1}, x_{2}, \ldots, x_{n} \in H$.

Since $f_{o}$ is additive, a long calculation yields

$$
\begin{aligned}
& D_{n} f_{o}\left(x_{1}, x_{2}, \ldots, x_{n}\right) \\
& \quad=\sum_{1 \leq i, j \leq n, i \neq j} A f_{o}\left(x_{i},-x_{j}\right)+2 \sum_{i=1}^{n-1} A f_{o}\left(\sum_{j=1}^{i} x_{j}, x_{i+1}\right) \\
& \quad=0 .
\end{aligned}
$$

Hence, it follows from (2.5) and (2.6) that

$$
D_{n} f\left(x_{1}, x_{2}, \ldots, x_{n}\right)=D_{n} f_{e}\left(x_{1}, x_{2}, \ldots, x_{n}\right)+D_{n} f_{o}\left(x_{1}, x_{2}, \ldots, x_{n}\right)=0
$$

for all $x_{1}, x_{2}, \ldots, x_{n} \in H$; that is, $f$ is a solution of (1.4).

\section{Generalized Hyers-Ulam Stability of (1.4)}

In the following theorem, we will investigate the stability problem of the functional equation (1.4).

Theorem 3.1. Assume that $n \geq 2$ is an integer. Let $H$ and $X$ be an additive group and a complete non-Archimedean space, respectively. Assume that $\varphi: H^{n} \rightarrow[0, \infty)$ is a function such that

$$
\lim _{m \rightarrow \infty} \frac{\varphi\left(n^{m} x_{1}, n^{m} x_{2}, \ldots, n^{m} x_{n}\right)}{|n|^{2 m}}=0
$$


for all $x_{1}, x_{2}, \ldots, x_{n} \in H$. Moreover, assume that the limit

$$
\tilde{\varphi}(x):=\lim _{m \rightarrow \infty} \max _{0 \leq i<m}\left\{\frac{\varphi\left(n^{i} x, \ldots, n^{i} x\right)}{|4||n|^{2 i+2}}, \frac{\varphi\left(-n^{i} x, \ldots,-n^{i} x\right)}{|4||n|^{2 i+2}}\right\}
$$

exists for each $x \in H$. If a function $f: H \rightarrow X$ satisfies the inequality

$$
\left\|D_{n} f\left(x_{1}, x_{2}, \ldots, x_{n}\right)\right\| \leq \varphi\left(x_{1}, x_{2}, \ldots, x_{n}\right)
$$

for any $x_{1}, x_{2}, \ldots, x_{n} \in H$, then there exists a unique quadratic-additive function $T: H \rightarrow X$ such that

$$
\|f(x)-T(x)\| \leq \tilde{\varphi}(x)
$$

for each $x \in H$. In particular, $T$ is given by

$$
T(x)=\lim _{m \rightarrow \infty}\left(\frac{f\left(n^{m} x\right)+f\left(-n^{m} x\right)}{2 n^{2 m}}+\frac{f\left(n^{m} x\right)-f\left(-n^{m} x\right)}{2 n^{m}}\right)
$$

for all $x \in H$.

Proof. If we replace $x_{i}$ in (3.1) with 0 for each $i \in\{1,2, \ldots, n\}$, then we have

$$
\lim _{m \rightarrow \infty} \frac{\varphi(0,0, \ldots, 0)}{|n|^{2 m}}=0
$$

Since $|n| \leq 1$, it holds that $\varphi(0,0, \ldots, 0)=0$ and

$$
\|(n-1)(n+2) f(0)\|=\left\|D_{n} f(0,0, \ldots, 0)\right\| \leq \varphi(0,0, \ldots, 0)=0 .
$$

Hence, we conclude that $f(0)=0$.

Let $J_{m} f: H \rightarrow Y$ be a function defined by

$$
J_{m} f(x)=\frac{f\left(n^{m} x\right)+f\left(-n^{m} x\right)}{2 n^{2 m}}+\frac{f\left(n^{m} x\right)-f\left(-n^{m} x\right)}{2 n^{m}}
$$


for all $x \in H$ and $m \in\{0,1,2, \ldots\}$. A tedious calculation, together with $\left(F_{2}\right),\left(N_{3}\right)$, and (3.3), yields

$$
\begin{aligned}
& \left\|J_{i} f(x)-J_{i+1} f(x)\right\|=\|-\frac{D_{n} f\left(n^{i} x, \ldots, n^{i} x\right)}{4 n^{2 i+2}}-\frac{D_{n} f\left(-n^{i} x, \ldots,-n^{i} x\right)}{4 n^{2 i+2}} \\
& -\frac{D_{n} f\left(n^{i} x, \ldots, n^{i} x\right)}{4 n^{i+1}}+\frac{D_{n} f\left(-n^{i} x, \ldots,-n^{i} x\right)}{4 n^{i+1}} \| \\
& \leq \max \left\{\frac{\left\|D_{n} f\left(n^{i} x, \ldots, n^{i} x\right)\right\|}{|4||n|^{2 i+2}}, \frac{\left\|D_{n} f\left(-n^{i} x, \ldots,-n^{i} x\right)\right\|}{|4||n|^{2 i+2}},\right. \\
& \left.\frac{\left\|D_{n} f\left(n^{i} x, \ldots, n^{i} x\right)\right\|}{|4||n|^{i+1}}, \frac{\left\|D_{n} f\left(-n^{i} x, \ldots,-n^{i} x\right)\right\|}{|4||n|^{i+1}}\right\} \\
& \leq \max \left\{\frac{\varphi\left(n^{i} x, \ldots, n^{i} x\right)}{|4||n|^{2 i+2}}, \frac{\varphi\left(-n^{i} x, \ldots,-n^{i} x\right)}{|4||n|^{2 i+2}}\right\}
\end{aligned}
$$

for all $x \in H$ and $i \in\{0,1,2, \ldots\}$. It follows from (3.1) and (3.9) that the sequence $\left\{J_{m} f(x)\right\}$ is Cauchy. Since $X$ is complete, we conclude that $\left\{J_{m} f(x)\right\}$ is convergent.

Let us define

$$
T(x):=\lim _{m \rightarrow \infty} J_{m} f(x)
$$

for any $x \in H$. It follows from $\left(N_{3}\right)$ and (3.9) that

$$
\begin{aligned}
\left\|f(x)-J_{m} f(x)\right\| & =\left\|\sum_{i=0}^{m-1}\left(J_{i} f(x)-J_{i+1} f(x)\right)\right\| \\
& \leq \max _{0 \leq i<m}\left\|J_{i} f(x)-J_{i+1} f(x)\right\| \\
& \leq \max _{0 \leq i<m}\left\{\frac{\varphi\left(n^{i} x, \ldots, n^{i} x\right)}{|4||n|^{2 i+2}}, \frac{\varphi\left(-n^{i} x, \ldots,-n^{i} x\right)}{|4||n|^{2 i+2}}\right\}
\end{aligned}
$$

for all $m \in\{0,1,2, \ldots\}$ and $x \in H$. In view of (3.2), if we let $m \rightarrow \infty$ in (3.11), then we obtain the inequality (3.4). get

Replacing $x_{i}$ in (3.3) with $n^{m} x_{i}$ for $i \in\{1,2, \ldots, n\}$ and considering $\left(F_{2}\right)$ and $\left(N_{3}\right)$, we

$$
\begin{aligned}
\left\|D_{n} J_{m} f\left(x_{1}, x_{2}, \ldots, x_{n}\right)\right\|=\| & \frac{D_{n} f\left(n^{m} x_{1}, \ldots, n^{m} x_{n}\right)-D_{n} f\left(-n^{m} x_{1}, \ldots,-n^{m} x_{n}\right)}{2 n^{m}} \\
& +\frac{D_{n} f\left(n^{m} x_{1}, \ldots, n^{m} x_{n}\right)+D_{n} f\left(-n^{m} x_{1}, \ldots,-n^{m} x_{n}\right)}{2 n^{2 m}} \|
\end{aligned}
$$


Abstract and Applied Analysis

$$
\begin{array}{r}
\leq \max \left\{\frac{\varphi\left(n^{m} x_{1}, \ldots, n^{m} x_{n}\right)}{|2||n|^{m}}, \frac{\varphi\left(-n^{m} x_{1}, \ldots,-n^{m} x_{n}\right)}{|2||n|^{m}},\right. \\
\left.\frac{\varphi\left(n^{m} x_{1}, \ldots, n^{m} x_{n}\right)}{|2||n|^{2 m}}, \frac{\varphi\left(-2^{m} x_{1}, \ldots,-2^{m} x_{n}\right)}{|2||n|^{2 m}}\right\}
\end{array}
$$

for all $m \in\{0,1,2, \ldots\}$ and $x_{1}, x_{2}, \ldots, x_{n} \in H$. If we let $m \rightarrow \infty$ in the last inequality, then it follows from the condition (3.1) that $D_{n} T\left(x_{1}, x_{2}, \ldots, x_{n}\right)=0$ for all $x_{1}, x_{2}, \ldots, x_{n} \in H$; that is, $T$ is a quadratic-additive function.

Assume that $T^{\prime}: H \rightarrow X$ is another quadratic-additive function satisfying (3.4). By the definition of $D_{n}$, a routine calculation yields

$$
\begin{aligned}
- & \frac{D_{n} T^{\prime}\left(n^{i} x, \ldots, n^{i} x\right)}{4 n^{2 i+2}}-\frac{D_{n} T^{\prime}\left(-n^{i} x, \ldots,-n^{i} x\right)}{4 n^{2 i+2}}-\frac{D_{n} T^{\prime}\left(n^{i} x, \ldots, n^{i} x\right)}{4 n^{i+1}}+\frac{D_{n} T^{\prime}\left(-n^{i} x, \ldots,-n^{i} x\right)}{4 n^{i+1}} \\
= & -\frac{1}{2 n^{2(i+1)}}\left(T^{\prime}\left(n^{i+1} x\right)+T^{\prime}\left(-n^{i+1} x\right)\right)+\frac{1}{2 n^{2 i}}\left(T^{\prime}\left(n^{i} x\right)+T^{\prime}\left(-n^{i} x\right)\right) \\
& -\frac{1}{2 n^{i+1}}\left(T^{\prime}\left(n^{i+1} x\right)-T^{\prime}\left(-n^{i+1} x\right)\right)+\frac{1}{2 n^{i}}\left(T^{\prime}\left(n^{i} x\right)-T^{\prime}\left(-n^{i} x\right)\right)
\end{aligned}
$$

for each $i \in\{0,1,2, \ldots\}$ and $x \in H$. Hence, it follows from (3.8) that

$$
\begin{aligned}
\sum_{i=0}^{k-1}( & -\frac{D_{n} T^{\prime}\left(n^{i} x, \ldots, n^{i} x\right)}{4 n^{2 i+2}}-\frac{D_{n} T^{\prime}\left(-n^{i} x, \ldots,-n^{i} x\right)}{4 n^{2 i+2}} \\
& \left.-\frac{D_{n} T^{\prime}\left(n^{i} x, \ldots, n^{i} x\right)}{4 n^{i+1}}+\frac{D_{n} T^{\prime}\left(-n^{i} x, \ldots,-n^{i} x\right)}{4 n^{i+1}}\right)=T^{\prime}(x)-J_{k} T^{\prime}(x)
\end{aligned}
$$

for any $k \in \mathbb{N}$ and $x \in H$. Since $T^{\prime}$ is a solution of (1.4), it follows from the last equality that

$$
T^{\prime}(x)=J_{k} T^{\prime}(x)
$$

for any $k \in \mathbb{N}$ and $x \in H$. Obviously, this equality also holds for $T$.

Consequently, by considering that $|n| \leq 1$, it follows from $\left(N_{3}\right),(3.1),(3.4)$, and (3.8) that

$$
\begin{aligned}
\| T(x) & -T^{\prime}(x) \| \\
\quad & \lim _{k \rightarrow \infty}\left\|J_{k} T(x)-J_{k} T^{\prime}(x)\right\| \\
& \leq \lim _{k \rightarrow \infty} \max \left\{\left\|J_{k} T(x)-J_{k} f(x)\right\|,\left\|J_{k} f(x)-J_{k} T^{\prime}(x)\right\|\right\}
\end{aligned}
$$




$$
\begin{aligned}
& \leq \lim _{k \rightarrow \infty}|2|^{-1}|n|^{-2 k} \max \left\{\left\|T\left(n^{k} x\right)-f\left(n^{k} x\right)\right\|,\left\|T\left(-n^{k} x\right)-f\left(-n^{k} x\right)\right\|,\right. \\
& \left.\qquad f\left(n^{k} x\right)-T^{\prime}\left(n^{k} x\right)\|,\| f\left(-n^{k} x\right)-T^{\prime}\left(-n^{k} x\right) \|\right\} \\
& \leq \lim _{k \rightarrow \infty} \lim _{m \rightarrow \infty} \max _{k \leq i<m+k}\left\{\frac{\varphi\left(n^{i} x, \ldots, n^{i} x\right)}{|8||n|^{2 i+2}}, \frac{\varphi\left(-n^{i} x, \ldots,-n^{i} x\right)}{|8 \| n|^{2 i+2}}\right\} \\
& =0
\end{aligned}
$$

for all $x \in H$. Therefore, $T=T^{\prime}$, which proves the uniqueness of $T$.

Corollary 3.2. Let $X$ and $Y$ be non-Archimedean normed spaces over $\mathbb{K}$ with $|n|<1$. If $Y$ is complete and $f: X \rightarrow Y$ satisfies the inequality

$$
\left\|D f\left(x_{1}, x_{2}, \ldots, x_{n}\right)\right\| \leq \theta \sum_{i=1}^{n}\left\|x_{i}\right\|^{r}
$$

for all $x_{1}, x_{2}, \ldots, x_{n} \in X$ and for some $r>2$, then there exists a unique quadratic-additive function $T: X \rightarrow Y$ such that

$$
\|f(x)-T(x)\| \leq \frac{n \theta}{|4||n|^{2}}\|x\|^{r}
$$

for all $x \in X$.

Proof. Let $\varphi\left(x_{1}, x_{2}, \ldots, x_{n}\right)=\theta \sum_{i=1}^{n}\left\|x_{i}\right\|^{r}$. Since $|n|<1$ and $r-2>0$, we get

$$
\lim _{m \rightarrow \infty}|n|^{-2 m} \varphi\left(n^{m} x_{1}, n^{m} x_{2}, \ldots, n^{m} x_{n}\right)=\lim _{m \rightarrow \infty}|n|^{m(r-2)} \varphi\left(x_{1}, x_{2}, \ldots, x_{n}\right)=0
$$

for all $x_{1}, x_{2}, \ldots, x_{n} \in X$. Therefore, the conditions of Theorem 3.1 are satisfied. Indeed, it is easy to see that $\tilde{\varphi}(x)=n \theta\left(|4|^{-1}|n|^{-2}\right)\|x\|^{r}$. By Theorem 3.1, there exists a unique quadraticadditive function $T: X \rightarrow Y$ such that (3.18) holds.

\section{Acknowledgments}

The second author was supported by the Basic Science Research Program through the National Research Foundation of Korea (NRF) funded by the Ministry of Education, Science and Technology (no. 2011-0004919).

\section{References}

[1] S. M. Ulam, A Collection of Mathematical Problems, Interscience Publishers, London, UK, 1960.

[2] D. H. Hyers, "On the stability of the linear functional equation," Proceedings of the National Academy of Sciences of the United States of America, vol. 27, pp. 222-224, 1941.

[3] T. Aoki, "On the stability of the linear transformation in Banach spaces," Journal of the Mathematical Society of Japan, vol. 2, pp. 64-66, 1950. 
[4] T. M. Rassias, "On the stability of the linear mapping in Banach spaces," Proceedings of the American Mathematical Society, vol. 72, no. 2, pp. 297-300, 1978.

[5] L. M. Arriola and W. A. Beyer, "Stability of the Cauchy functional equation over $p$-adic fields," Real Analysis Exchange, vol. 31, no. 1, pp. 125-132, 2006.

[6] I.-S. Chang, E. H. Lee, and H.-M. Kim, "On Hyers-Ulam-Rassias stability of a quadratic functional equation," Mathematical Inequalities \& Applications, vol. 6, no. 1, pp. 87-95, 2003.

[7] S. Czerwik, Functional Equations and Inequalities in Several Variables, World Scientific Publishing, River Edge, NJ, USA, 2002.

[8] A. Ebadian, N. Ghobadipour, Th. M. Rassias, and M. Eshaghi Gordji, "Functional inequalities associated with Cauchy additive functional equation in non-Archimedean spaces," Discrete Dynamics in Nature and Society, vol. 2011, Article ID 929824, 14 pages, 2011.

[9] P. Găvruța, "A generalization of the Hyers-Ulam-Rassias stability of approximately additive mappings," Journal of Mathematical Analysis and Applications, vol. 184, no. 3, pp. 431-436, 1994.

[10] M. Eshaghi Gordji, "Nearly ring homomorphisms and nearly ring derivations on non-Archimedean Banach algebras," Abstract and Applied Analysis, vol. 2010, Article ID 393247, 12 pages, 2010.

[11] M. Eshaghi Gordji and M. B. Savadkouhi, "Stability of a mixed type cubic-quartic functional equation in non-Archimedean spaces," Applied Mathematics Letters, vol. 23, no. 10, pp. 1198-1202, 2010.

[12] M. Eshaghi Gordji and M. B. Savadkouhi, "Stability of cubic and quartic functional equations in nonArchimedean spaces," Acta Applicandae Mathematicae, vol. 110, no. 3, pp. 1321-1329, 2010

[13] D. H. Hyers, G. Isac, and T. M. Rassias, Stability of Functional Equations in Several Variables, Birkhäuser Boston Inc., Boston, Mass, USA, 1998.

[14] K.-W. Jun and Y.-H. Lee, "A generalization of the Hyers-Ulam-Rassias stability of the Pexiderized quadratic equations-II," Kyungpook Mathematical Journal, vol. 47, no. 1, pp. 91-103, 2007.

[15] S.-M. Jung, "Hyers-Ulam-Rassias stability of Jensen's equation and its application," Proceedings of the American Mathematical Society, vol. 126, no. 11, pp. 3137-3143, 1998.

[16] S.-M. Jung, Hyers-Ulam-Rassias Stability of Functional Equations in Nonlinear Analysis, vol. 48, Springer, New York, NY, USA, 2011.

[17] G. H. Kim, "On the stability of functional equations with square-symmetric operation," Mathematical Inequalities E Applications, vol. 4, no. 2, pp. 257-266, 2001.

[18] H.-M. Kim, "On the stability problem for a mixed type of quartic and quadratic functional equation," Journal of Mathematical Analysis and Applications, vol. 324, no. 1, pp. 358-372, 2006.

[19] Y.-H. Lee, "On the stability of the monomial functional equation," Bulletin of the Korean Mathematical Society, vol. 45, no. 2, pp. 397-403, 2008.

[20] Y.-H. Lee and K.-W. Jun, "A generalization of the Hyers-Ulam-Rassias stability of Jensen's equation," Journal of Mathematical Analysis and Applications, vol. 238, no. 1, pp. 305-315, 1999.

[21] Y.-H. Lee and K.-W. Jun, "A generalization of the Hyers-Ulam-Rassias stability of the Pexider equation," Journal of Mathematical Analysis and Applications, vol. 246, no. 2, pp. 627-638, 2000.

[22] A. K. Mirmostafaee, "Approximately additive mappings in non-Archimedean normed spaces," Bulletin of the Korean Mathematical Society, vol. 46, no. 2, pp. 387-400, 2009.

[23] F. Skof, "Local properties and approximation of operators," Rendiconti del Seminario Matematico e Fisico di Milano, vol. 53, pp. 113-129, 1983.

[24] M. S. Moslehian and T. M. Rassias, "Stability of functional equations in non-Archimedean spaces," Applicable Analysis and Discrete Mathematics, vol. 1, no. 2, pp. 325-334, 2007.

[25] W. Towanlong and P. Nakmahachalasint, "An n-dimensional mixed-type additive and quadratic functional equation and its stability," Science Asia, vol. 35, pp. 381-385, 2009. 


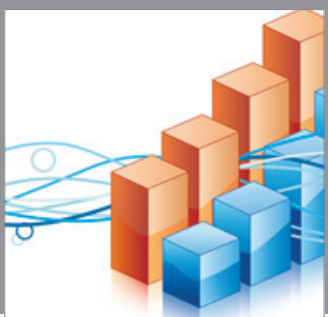

Advances in

Operations Research

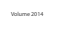

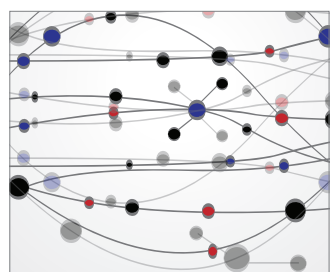

\section{The Scientific} World Journal
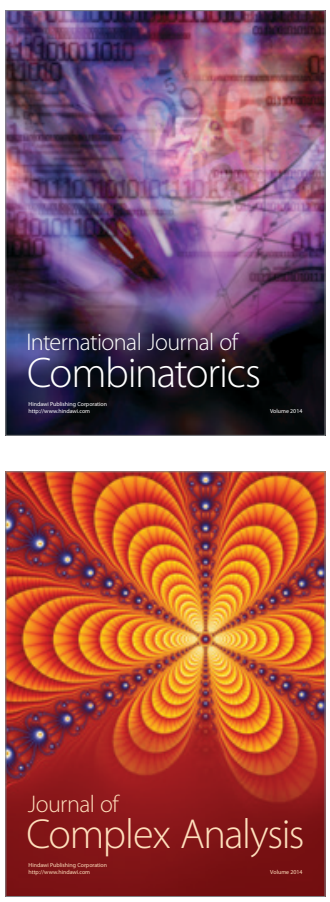

International Journal of

Mathematics and

Mathematical

Sciences
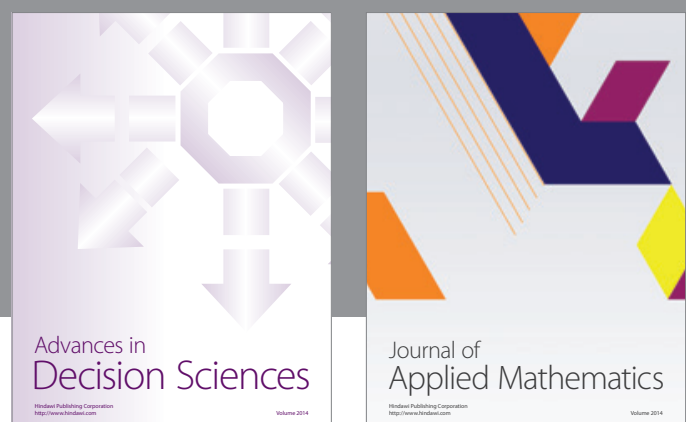

Journal of

Applied Mathematics
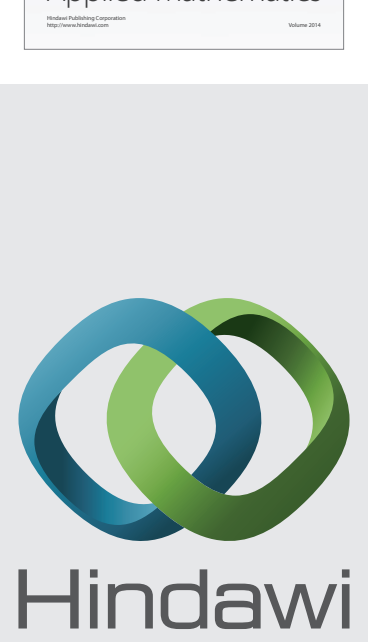

Submit your manuscripts at http://www.hindawi.com
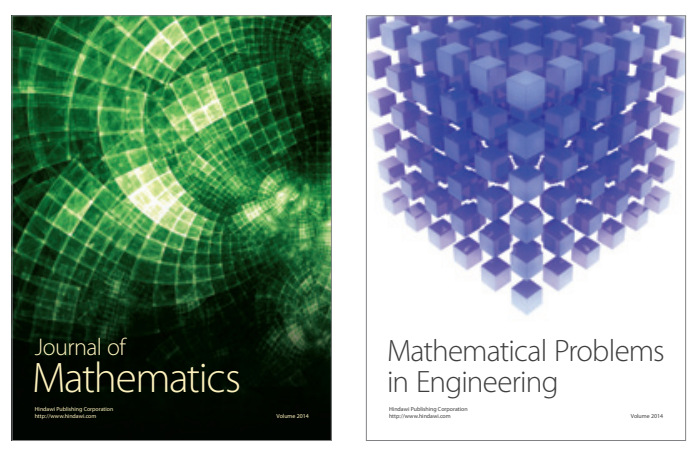

Mathematical Problems in Engineering
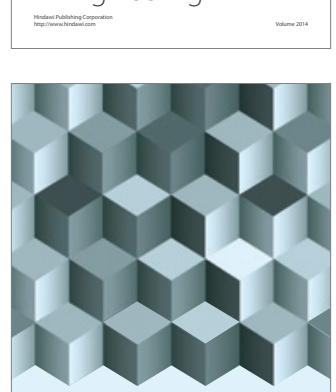

Journal of

Function Spaces
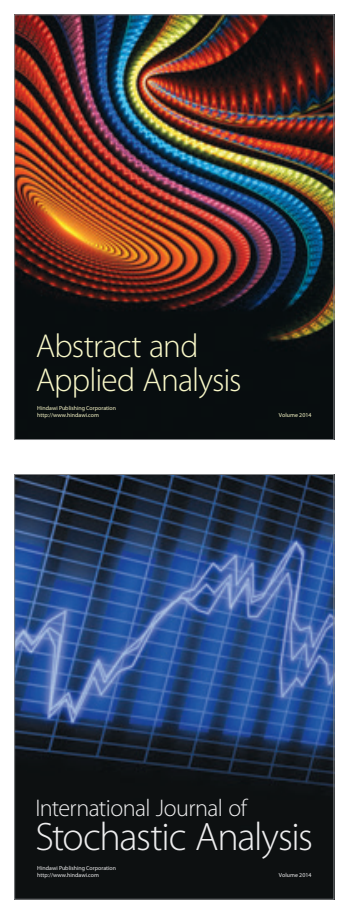

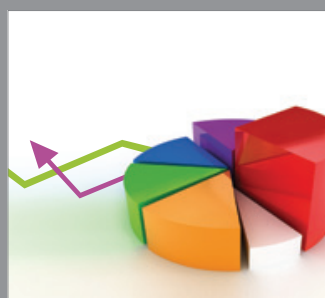

ournal of

Probability and Statistics

Promensencen
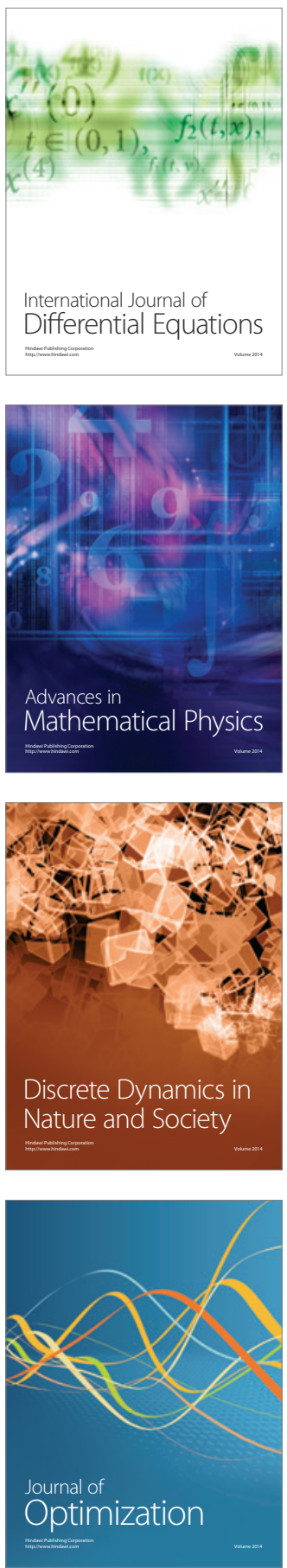